\title{
Temporal Stability of High-Frequency Brain Oscillations in the Human EEG
}

\author{
Andreas Keil*, Margarita Stolarova*, Sabine Heim*, Thomas Gruber+, and Matthias M. Müller+
}

\begin{abstract}
Summary: Temporal stability of a given measurement within individual participants is a desirable property of psychophysiological measures. The present study aims to examine the reliability of high-frequency oscillatory activity in the human electroencephalogram (EEG) across 4 sessions. Convolution of the EEG time series with Morlet wavelets yielded time-frequency representations of the signal for each session. Stability of both topography and time course of gamma-band activity (GBA) was determined for two participants performing a feature-based selective attention task in four separate sessions, spaced at weekly intervals. We found high temporal stability of non-phase-locked GBA typically occurring in time ranges between 200 and 500 ms following presentation of a stimulus, both in terms of topography and time course. Early phase-locked GBA (80-120 ms) showed higher variability with respect to topography, but was consistent in terms of time course. We conclude that measures of high-frequency oscillatory activity as used in the cognitive neurosciences meet stability requirements necessary for meaningful interpretation of this parameter of brain function.
\end{abstract}

Key words: Oscillatory brain activity; EEG; Visual perception; Reliability.

\section{Introduction}

Oscillatory activity in the human electroencephalogram (EEG) has been used as a dependent variable in numerous experimental paradigms tapping inter alia perceptual integration, attention, memory, emotion, and motivation (Sannita 2000; Tallon-Baudry et al. 1999). A substantial body of this research has focused on gamma band activity (GBA), which can be measured as amplitude and coherence changes in the upper range of the temporal spectrum, typically above $20 \mathrm{~Hz}$. While spectral power in lower frequency bands has been established as a reliable measure of brain functioning (Basar et al. 2001), information concerning the reliability of high-frequency phenomena is scarce.

As known from animal work, oscillatory neuronal responses may be non-phase- and time-locked to the onset of a stimulus across repetitions of the same token (Tallon-Baudry and Bertrand 1999). Therefore, analysis techniques must account for possible inter-trial jitter

* Department of Psychology, University of Konstanz, Germany.

+ Department of Psychology, University of Leipzig, Germany.

Accepted for publication: September 29, 2003.

Research was supported by the Deutsche Forschungsgemeinschaft.

Correspondence and reprint requests should be addressed to Andreas Keil, Department of Psychology, University of Konstanz, Box D23, D-78434 Konstanz.

Fax: +49 7531882891

E-mail: Andreas.Keil@uni-konstanz.de
(Bertrand et al. 1994). Furthermore, to obtain meaningful parameters of GBA, the problems of noise, small signal amplitudes, and brevity of GBA bursts must be considered, thus constituting a challenge for pre-processing and artifact control methods (Hauk et al. 2002).

One approach that has repeatedly been applied to meet these requirements is the time-frequency (TF) representation of the signal (e.g. Gruber et al. 2002; Keil et al. 1999). TF representations can be computed by convolution of EEG time series with a wavelet family, resulting in the time-varying energy for each frequency band (evolutionary spectrum) at near-optimal resolution in the time and frequency domain for the frequency range of interest (Bertrand et al. 1994). This method has been used successfully in studies of visual perception and has revealed two main features of the visual high-frequency response in the human EEG: (1) an early, time and phase-locked response following $80-150 \mathrm{~ms}$ the onset of a stimulus, which is most pronounced in frequency ranges between 20 and $50 \mathrm{~Hz}$, and (2) a non-phase-locked response in a frequency range above $40 \mathrm{~Hz}$, often seen in a time window between 200 and $500 \mathrm{~ms}$ after stimulus onset (Müller et al. 2000). The two types of spectral events have been shown to correlate with distinct aspects of the experimental manipulation: in terms of early evoked gamma, Herrmann and colleagues (1999) reported frontal GBA responses during target detection that were greatest when stimulus features were task-relevant. These authors concluded that frontal GBA is associated with top-down processes, comparing incoming information with templates in working memory. Likewise, 
auditory targets were effective in enhancing phase-locked GBA over frontal leads, when paired with a motor task (Yordanova et al. 2001). There, sensory-motor integration was proposed as a possible function of neuronal assemblies encompassing the prefrontal cortex. In contrast, late induced GBA has often been shown to vary as a function of attention, perceptual coherence, or gestalt properties (Keil et al. 2001a). Theoretically, these modulations of induced GBA have been related to bottom-up processing of a stimulus representation, or alternatively, to the activation of a network representing aspects of the stimulus (e.g. Gruber et al. 2002) as well as behavioral dispositions and memory traces (Keil et al. 2001c; Slobounov et al. 2000).

It is still a matter of debate if changes in high-frequency spectral power reflect meaningful neural processes (Keil et al. 2001a). For instance, the detection of GBA from human EEG recordings may require different tasks and experimental paradigms, compared to animal work (Juergens et al. 1999). Reliability in the sense of temporal stability represents one important property of meaningful measures. To examine stability of the two brain responses mentioned above, we obtained evolutionary spectra of EEG recorded during a feature-based attention task in two participants, across four subsequent recording sessions. Thus, our aim was not to show whether or not re-test reliability of GBA was high or low in a large sample of subjects, but rather we sought to determine GBA stability within a given participant across multiple measurements. We hypothesized that both the early evoked response and the induced higher-frequency GBA in the later time window show a high degree of stability in terms of latency, frequency range, and topography.

\section{Methods}

\section{Participants}

After approval by our ethics board, two male participants (ages 27 and 32) were recruited from the subject pool of an earlier study on GBA during emotional picture perception. Their data had high signal-to-noise ratio and a low number of artifact-contaminated EEG epochs. The participants evidenced normal vision and were recorded in 4 sessions spaced at 7 to 9 day intervals, respectively. They received 40 Euros for participating.

\section{Stimuli and Procedure}

Colored checkerboard stimuli were used for the feature-based attention task. Standard stimuli $(\mathrm{p}=0.80)$ were green-and-gray and red-and-gray $7 \times 7$ isoluminant checkerboards subtending $4.0 \times 4.0 \mathrm{deg}$. of visual angle. Rare target stimuli $(p=0.20)$ were designed as checkerboards with a different hue in green and red, respectively, targets being brighter $(9.7 \mathrm{~cd} / \mathrm{sqm})$ as compared to non-targets $(6.3 \mathrm{~cd} / \mathrm{sqm})$. A central white fixation cross was always present. All stimuli were shown centrally on a 21 inch monitor, situated $1.5 \mathrm{~m}$ in front of the subjects. Experimental trials comprised presentation of a checkerboard for $200 \mathrm{~ms}$, followed by variable inter-stimulus intervals ranging between 1000 to 1500 ms during which the participants were asked to respond to target stimuli as fast and as accurate as possible. Target and standard checkerboards were presented in randomized order.

Participants attended either to bright green or bright red targets and pressed the space bar of the computer keyboard accordingly. This resulted in 2 experimental conditions (attend bright green vs. attend bright red). These conditions were organized into 4 blocks ( 2 blocks "attend red" and 2 blocks "attend green") having 288 trials each. Each block lasted approximately $16 \mathrm{~min}$. The sequence of blocks was counterbalanced across the participants. Likewise, the responding hand was changed half way through the experiment, and the sequence of hand usage was counterbalanced across the two subjects. Practice trials were provided for each subject and condition to ensure that they were able to perform the task correctly.

\section{Data Acquisition and Analysis}

EEG was recorded continuously using an Electrical Geodesics 128-electrode array (Electric Geodesic, Inc., EGI, Eugene, Oregon, USA). Site Cz was used as the recording reference. As recommended for the EGI high input impedance amplifier, electrode impedances were kept below $50 \mathrm{kOhms}$. Data were digitized at $500 \mathrm{~Hz}$ and all channels were preprocessed on-line by means of a 0.1 to $200 \mathrm{~Hz}$ band-pass filter. Vertical and horizontal eye movements were monitored by means of a subset of the 128 electrodes. Further data processing was performed off-line. The average reference was used for all analyses described below.

Data analyses focused on brain responses to standard stimuli sharing color with the target stimulus. This approach (i) excluded effects of the motor response to targets and (ii) enclosed stimuli with task relevance, being viewed more attentively than non-attended color standard stimuli. Epochs of $1024 \mathrm{~ms}$ length (300 ms before and $724 \mathrm{~ms}$ after stimulus onset) were extracted. These segments were artifact corrected using a procedure proposed by Junghöfer and co-workers (statistical correction of artifacts in dense array studies, SCADS, 2000). This procedure consists of a combination of trial exclusion and channel approximation based on statistical parameters of the data. The information of eliminated electrodes is replaced with a statistically weighted spherical interpolation from the full channel set. The limit for 
the number of approximated channels was set to 20 channels. With respect to the spatial arrangement of the approximated sensors, it was ensured that the rejected sensors were not located within one region of the scalp, as this would make interpolation for this area invalid. Single epochs with excessive eye-movements and blinks or more than 20 channels containing artifacts were discarded. The average rejection rate across sessions and the two participants was $19 \%$ for standard stimuli.

\section{Time-Frequency Analysis}

Time-varying spectra of EEG epochs were obtained by convoluting EEG time series with complex Morlet wavelets, which overcomes problems associated with constant Fourier window length as used in spectrogram approaches (Sinkkonen et al. 1995). Morlet wavelets (g) can easily be generated in the time domain for different analysis frequencies $f_{0}$ according to equation 1 .

$$
g\left(t, f_{0}\right)=A^{\prime} e^{\frac{t^{2}}{2 \sigma_{t}^{2}}} e^{2 i \pi f_{0} t}
$$

with $A^{\prime}$ depending on the parameter $\boldsymbol{\sigma}_{f}$, specifying the width of the wavelet in the frequency domain, the analysis frequency $f_{0}$ and the user-selected ratio $m$ :

$$
A^{\prime}=\boldsymbol{\sigma}_{f} \sqrt{2 \pi^{3}} \sqrt{\frac{m}{f_{0} \sqrt{\pi}}}
$$

with

$$
m=\frac{f_{0}}{\boldsymbol{\sigma}_{f}}
$$

As a consequence, using a constant ratio $m$, the width of the wavelets in the frequency domain $\sigma_{f}$ changes as a function of the analysis frequency $f_{0}$. Time resolution for upper frequencies is superior compared to low frequency ranges, where frequency resolution is high, but time resolution is coarse. Thus, wavelet analysis is well-suited for detecting high-frequency oscillations that may occur in short bursts (Bertrand et al. 1994). Further methodological aspects of this approach are illustrated in Tallon-Baudry and Bertrand (1999). In the present study, we selected a constant ratio between a wavelet's frequency $f 0$ and the standard deviation of the wavelet in the frequency domain $\sigma_{\mathrm{f}}$, resulting in $\mathrm{f} 0 / \sigma_{\mathrm{f}}=7$. This was done in order to achieve good time and frequency resolution for $f 0$ ranging from 9.77 to $70.34 \mathrm{~Hz}$ in $0.49 \mathrm{~Hz}$ steps, yielding a total of 125 wavelets. For artifact-free EEG epochs (see figure 1), time-varying energy in a given frequency band was obtained as the squared absolute value of the convolution of the cosine-square-tapered signal with the wavelet. Single trial time-by-frequency matrices were averaged in order to obtain the evolutionary spectrum for each electrode and experimental condition. Phase-locking of oscillatory activity was measured using the phase-locking factor (R) as described by Tallon-Baudry and collaborators (1997). This measure of phase identity across trials can be obtained by means of phase-averaging across trials and is bounded between 0 (non-phase-locked signal) and 1 (phase-locked signal). Its statistical significance can be assessed by means of a circular statistic (Rayleigh test).

The mean spectral power for each sensor and wavelet within an epoch ranging from 280 to $100 \mathrm{~ms}$ prior to stimulus onset was subtracted to correct for baseline offset. TF representations of attended standard stimuli (sharing color, but not brightness with the target) were separately averaged across all electrode sites posterior to the vertex, resulting in a mean evolutionary spectrum for each subject and session. Electrode sites used for these TF plots were selected on the basis of previous findings regarding visual information processing (Gruber et al. 1999; Keil et al. 2001b; Keil et al. 1999; Müller et al. 2000; Tallon-Baudry and Bertrand 1999).

\section{Statistical Analysis}

Stability of GBA was assessed with respect to the topographical and temporal aspects of the response. To examine stability of GBA topographies across sessions, a commonly used approach in human GBA research was employed: Early and late high-frequency oscillations were separately analyzed as the mean spectral power in two time and frequency ranges. Early GBA was determined as the mean spectral power in a 21 to $32 \mathrm{~Hz}$ range between 80 and $120 \mathrm{~ms}$ post-stimulus; late GBA was defined in a 45 to $70 \mathrm{~Hz}$ range between 250 and $500 \mathrm{~ms}$ post-stimulus. Figure 2 demonstrates that these time-frequency windows contained meaningful information across recording sessions and participants.

For statistical analysis, vectors containing the mean spectral power in these time-frequency ranges at each sensor were calculated, representing the individual GBA topography for subjects and recording sessions. Thus, for each time-frequency range, participant, and recording session, one 129-element vector was obtained. Stability of topographies across sessions was then evaluated by means of Pearson correlation coefficients, using electrodes as cases and sessions as variables. This was conducted for each participant and time-frequency range separately.

To assess stability of GBA time course, a similar approach was used. The post-stimulus segment of the time-frequency representation $(0$ to $600 \mathrm{~ms}$ post-stimulus, i.e. 300 sample points) was averaged within the same fre- 


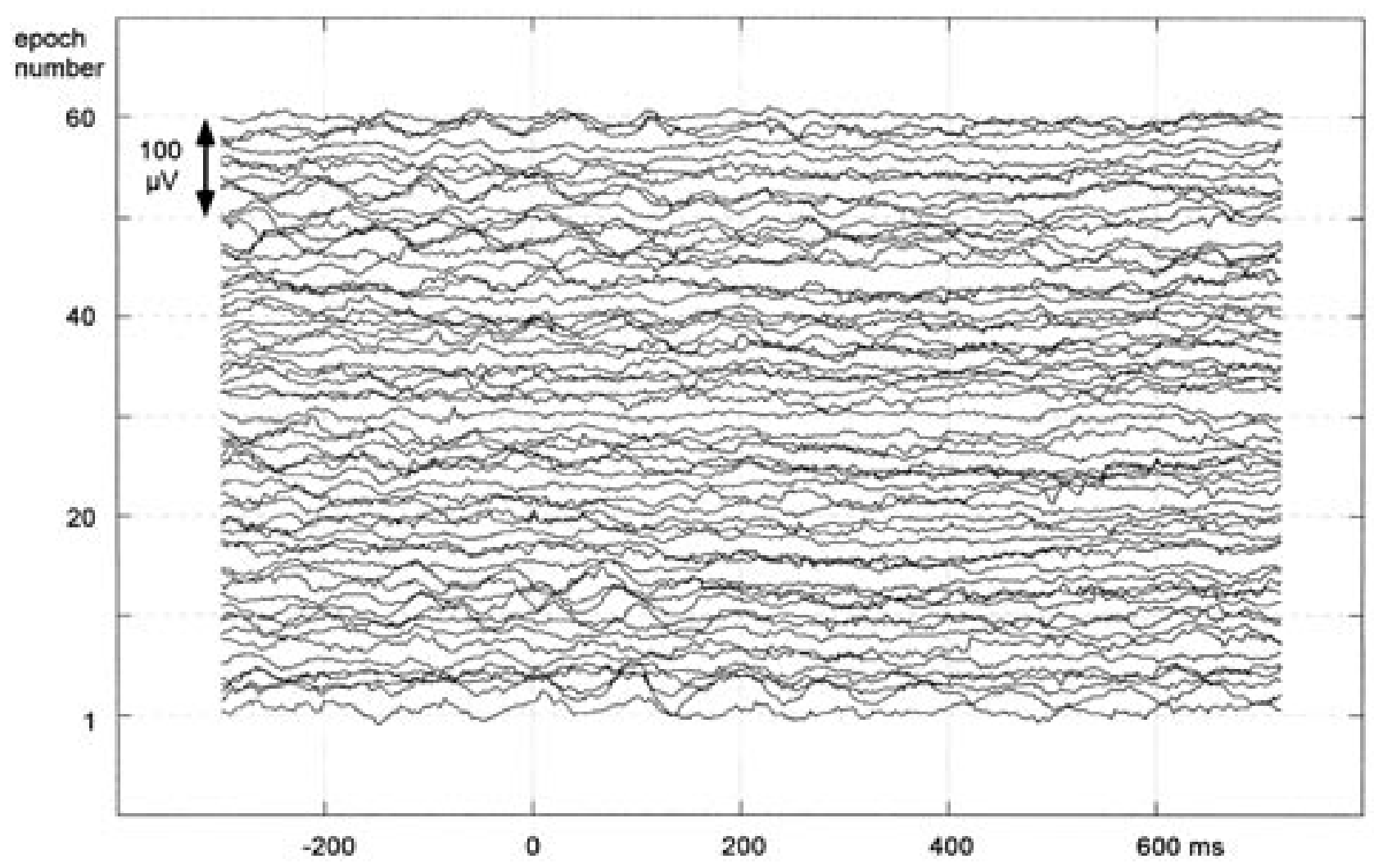

Figure 1. Subset of artifact-free EEG epochs (1-60) recorded from participant 1 at electrode site Poz (online band-pass-filtered at 0.1-100 Hz) during presentation of attended standard stimuli (sharing color, but not brightness with the target). Note that low-frequency activity is reduced following stimulus onset (time point zero), and high-frequency activity increases. Horizontal grids are spaced at $100 \mu \mathrm{V}$.

quency ranges as used for topographical analysis (see above). Thus, vectors were obtained reflecting temporal development of power in the two GBA bands across time. These time series were averaged across all sensors posterior to site $\mathrm{Cz}$ of the international 10-20 system. Separate 300-element vectors were calculated for each participant, frequency range, and recording session. Subsequently correlation analyses were performed using Pearson correlation coefficients. To this end, time points were used as cases, and sessions were used as variables. As indicated in the results section, this procedure resulted in measures of linear statistical relationship between frequency-specific gamma time series across sessions, for each participant.

\section{Results}

\section{Behavioral Data}

The two subjects performed the target detection task with sufficient accuracy. Their mean hit rates were 82.6 and 91.2 per cent. Reaction times for correct responses were 493 and $512 \mathrm{~ms}$, respectively. In terms of inter-session effects, both participants showed linear improvement in hit rate from session 1 to session 4 . No such effect was observed for reaction time. False alarms defined as a button press to standard stimuli were shown in 2.2 and 1.6 per cent of the standard trials, respectively.

\section{Spectral Data}

Phase-Locked Gamma (21-32 Hz)

Both subjects showed early phase-locked GBA enhancement in the time range selected for analysis, i.e. between 80 and $120 \mathrm{~ms}$ after stimulus onset. Maximum phase-locking factors in this time-frequency range were .41 and .53 for subject 1 and 2, respectively, and were highly significant (Rayleigh tests, ps < .01). Indices of topographical stability across the four sessions are presented in table I (top). Subject 1 (above diagonal) showed low positive correlations for the first sessions $(\mathrm{r}=.22, \mathrm{p}<0.09)$, decreasing to zero correlations for sessions 3 and 4 . None of these values reached statistical significance. For subject 2 (below diagonal) correlations were high for subsequent sessions (rs > .79; ps $<0.01$, see figure 3), and moderately decreased for 


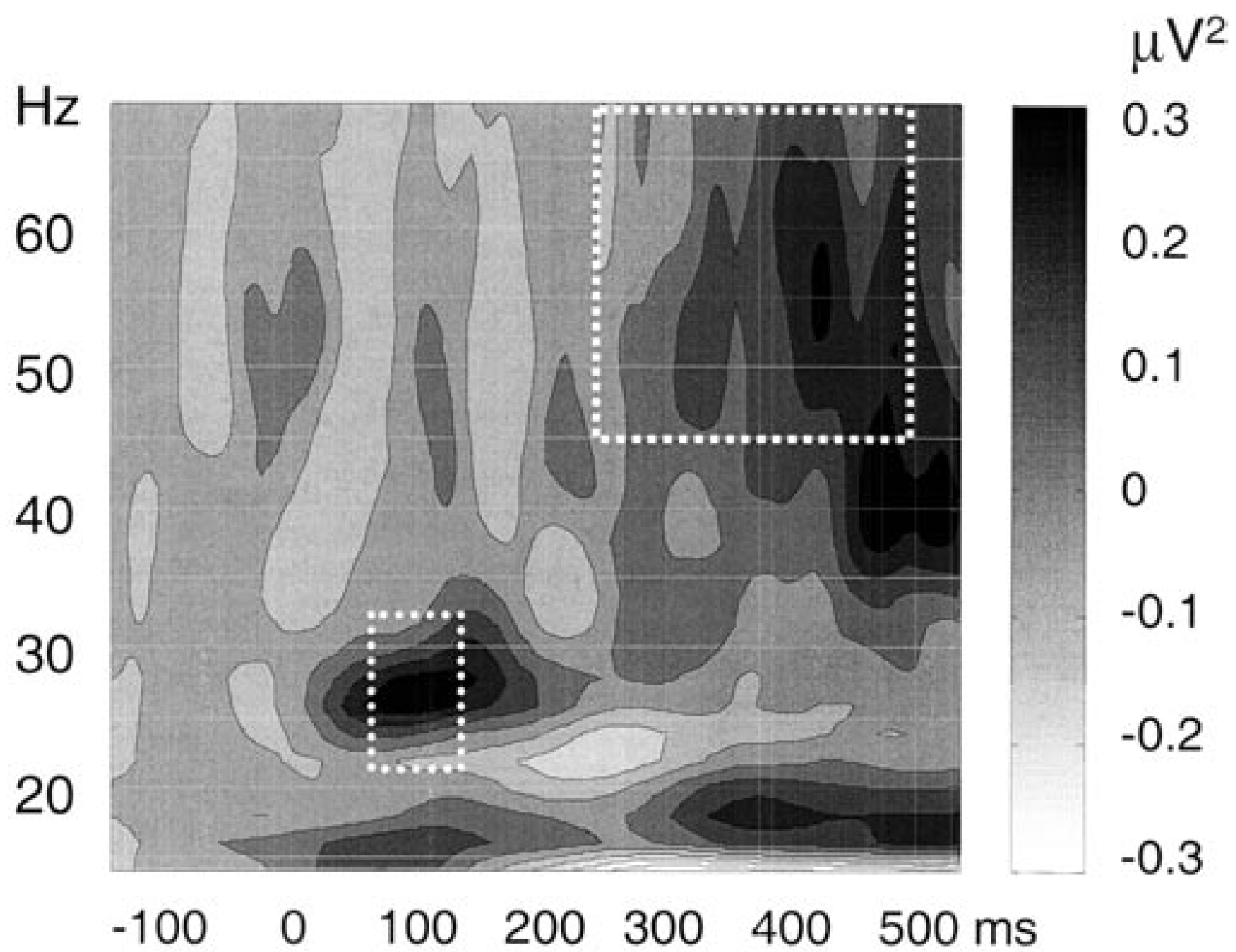

Figure 2. Mean baseline-corrected time-frequency representation of EEG epochs recorded during presentation of attended standard stimuli (sharing color, but not brightness with the target). Values were averaged across all electrode sites posterior to the vertex, and across four sessions and two participants. Time-frequency ranges selected for correlation analyses are shown as white rectangles.

the remaining sessions. As expected, correlations between subsequent recordings were higher than for sessions being separated by longer intervals, in both participants.

Time course of phase-locked GBA modulations at posterior sensors (see table I) displayed higher stability, compared to topography. Time series obtained from subject 1 (above diagonal) were reliable particularly among sessions 2,3 , and 4 (ps $<.05$ ), whereas the first session showed a significant relationship with session 4 only $(\mathrm{p}<$ .05). Subject 2 (below diagonal) again displayed higher stability across sessions than subject $1 \mathrm{did}$. All correlations between subsequent recording sessions reached statistical significance $(\mathrm{rs}<.70 ; \mathrm{ps}<.01)$. Furthermore, sessions 1 and 3 were systematically related $(\mathrm{r}=.66 ; \mathrm{p}<.01)$.

\section{Induced Gamma $(45-70 \mathrm{~Hz})$}

Figure 4 depicts the topographical distribution of induced GBA ( 250 to $500 \mathrm{~ms}$ post-stimulus) for all recording sessions in subject 1 . This late GBA enhancement was not phase-locked to the onset of checkerboard stimuli, as reflected in low phase-locking indices that failed to reach significance at any point located in the relevant time-frequency range. Induced GBA measured from this subject showed significant stability throughout (ps $<.05$, see table I, third tier, above diagonal). As can be seen from figure 4, induced GBA showed a right-hemisphere preponderance in session 1 only, whereas topographies in the other sessions had maxima at sites on the left hemisphere. For subject 2 (see table I, third tier, below diago- 
Table I. Between-session Pearson correlation coefficients for variables representing phase-locked GBA topographies (first tier), phase-locked GBA time series (second tier), induced GBA topographies (third tier), and induced GBA time series (fourth tier), recorded in the four sessions. Time series encompass 300 time points, topographies include 129 electrodes each. For each variable, coefficients are shown for subject 1 (above diagonal) and subject 2 (below diagonal).

\begin{tabular}{|c|c|c|c|c|}
\hline & Session 1 & Session 2 & Session 3 & Session 4 \\
\hline Session 1 & --- & .22 & .12 & .09 \\
\hline Session 3 & .85 & .87 & --- & -.15 \\
\hline \multirow[t]{2}{*}{ Session 4} & .37 & .38 & .79 & --- \\
\hline & \multicolumn{4}{|c|}{ Phase-locked GBA time series } \\
\hline Session 1 & -- & .29 & .18 & .53 \\
\hline Session 2 & .98 & --- & .64 & .46 \\
\hline Session 3 & .66 & .70 & -- & .73 \\
\hline Session 1 & --- & .48 & .61 & .47 \\
\hline Session 2 & .85 & --- & .57 & .71 \\
\hline Session 3 & .54 & .77 & -- & .80 \\
\hline \multirow[t]{2}{*}{ Session 4} & .03 & .25 & .81 & --- \\
\hline & \multicolumn{4}{|c|}{ Induced GBA time series } \\
\hline Session 1 & -- & .80 & .63 & .67 \\
\hline Session 2 & .89 & --- & .82 & .80 \\
\hline
\end{tabular}

nal), significant linear relationships between subsequent sessions ( $\mathrm{rs}>.77$, ps $<.01$ ) were observed. These decreased linearly as a function of inter-session distance, resulting in a zero correlation between topographies obtained in sessions 1 and 4.

The time course of induced GBA exhibited a high degree of stability throughout (see table I, bottom). For subject 1 , all correlations between subsequent sessions reached significance ( $\mathrm{rs}>.78$; $\mathrm{ps}<.01)$, and the same pattern emerged for subject 2 (rs $>.88$; ps < .01). Furthermore, the linear relationship between the other sessions was also significant in both participants (rs > .40; ps < .05). This characteristic of GBA time course is also illustrated in figure 5 .

\section{Discussion}

Temporal stability of dependent variables in an experimental context is one requirement for meaningful parameters used in the cognitive neurosciences. The present study aimed at examining measures of GBA in the human EEG as to their reliability across recording sessions in two healthy participants. Our analysis reveals that important characteristics of high-frequency activity in human EEG can reliably be observed within a subject when measurements are repeated. Both participants' evolutionary spectrum contained a phase-locked, lower frequency gamma response in early time segments as well as a late, induced GBA enhancement in higher 


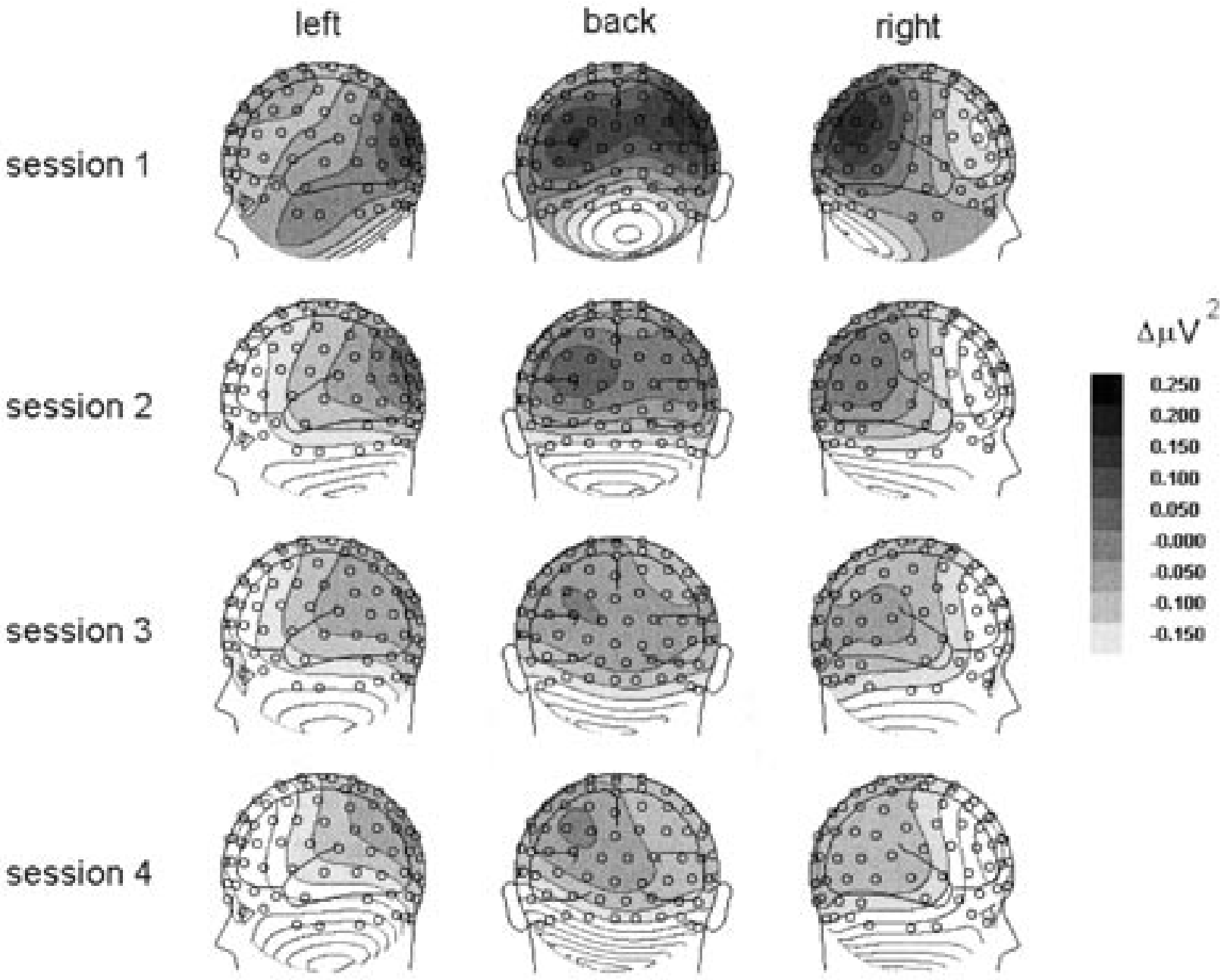

Figure 3. Mean baseline-corrected topographies of early evoked EEG spectral power (21-32 Hz), averaged across time points between 80 and 120 ms post-stimulus. Topographical distributions obtained from subject 2 are drawn from left, back, and right views for each of the four sessions.

ranges of the spectrum. These major components of high-frequency oscillatory activity occurred during specific time windows, which could easily be identified across experimental sessions.

Specifically, the early phase-locked component was most pronounced in a time range between 80 and $120 \mathrm{~ms}$, while late GBA showed its peak at about 200-500 ms. These time windows are consistent with previous reports on time-frequency analyses of EEG during visual perception (Müller et al. 2000; Sannita 2000; Tallon-Baudry and Bertrand 1999). Topography and time course of induced GBA changes were associated with higher stability across the two subjects, compared to evoked GBA. The latter response did not show topographical stability in subject 1 , whose correlation coefficients were smaller than $r=.22$. In subject 2, however, we observed high stability for phase-locked GBA topography, pointing to inter-individual variability in the occurrence of this high-frequency response. It is conceivable that this variability reflects problems of fixed-window approaches to data reduction in the time-frequency plane. Task-dependent changes in frequency or latency may go undetected with a fixed window such as used in the present study. It is thus mandatory to select these windows on the basis of time-frequency distributions for individual participants and conditions. In the present set of data, there was however no evidence for systematic session-dependent modulation of GBA center frequency or latency, respectively.

A homogeneous pattern was observed for the induced GBA enhancement in the range between 250 and $500 \mathrm{~ms}$ post-stimulus. Both topography and time course showed satisfactory stability across sessions. As can be 


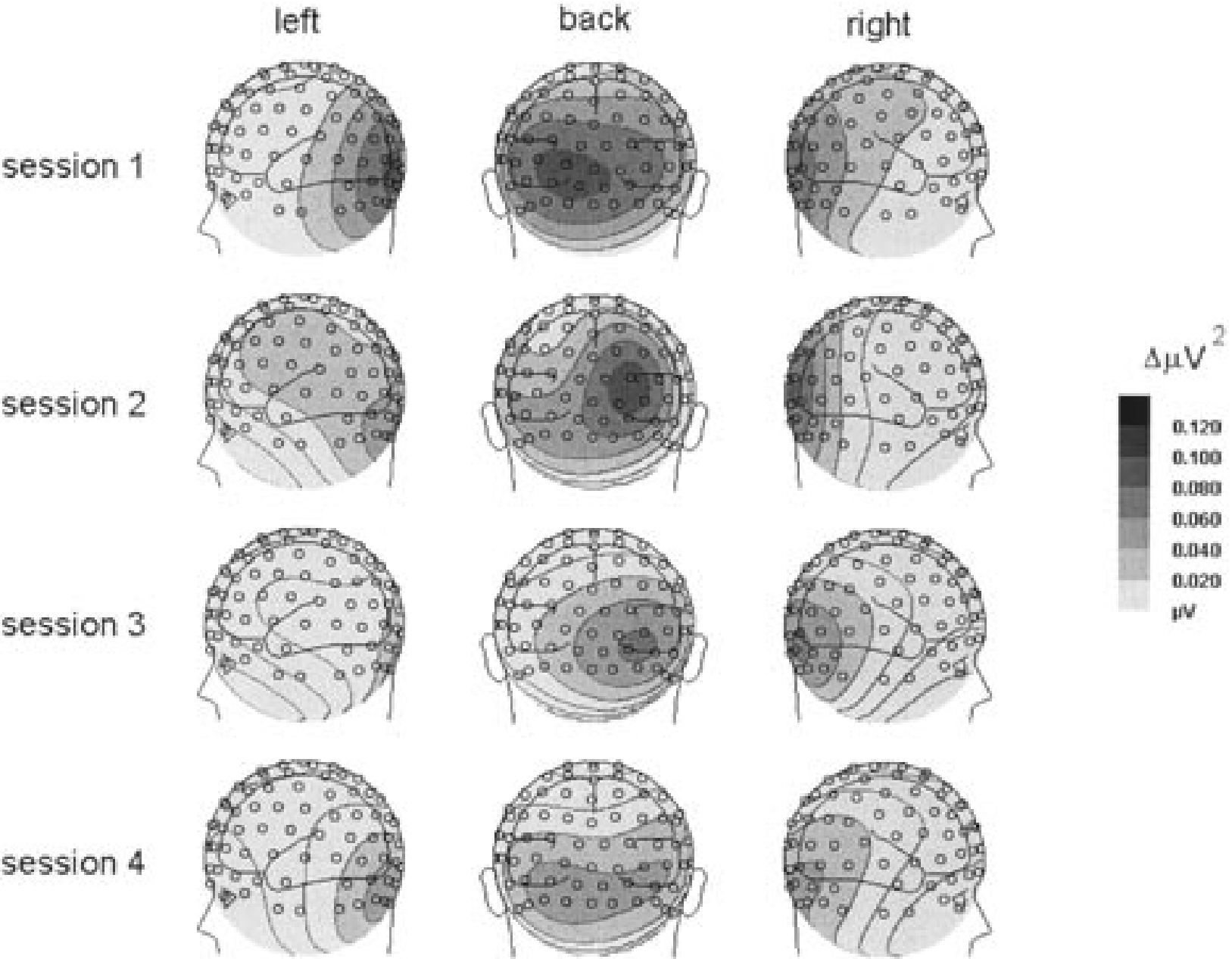

Figure 4. Mean baseline-corrected topographies of late induced EEG spectral power $(45-70 \mathrm{~Hz})$, averaged across time points between 250 and 500 ms post-stimulus. Topographies obtained from subject 1 are shown from left, back, and right views for each of the four sessions.

expected, greater temporal distance between sessions was associated with lower correspondence between parameters of oscillatory activity, possibly reflecting changes of familiarity with the task and experimental environment as well as effects of practice.

Interestingly, the magnitude of the two major time-frequency responses observed in the data set varied consistently as a function of time. The amplitude of late induced GBA gradually decreased across sessions, probably reflecting effects of habituation and/or repetition that have previously been observed in repeated measurement of electrophysiological or functional imaging parameters (Desimone 1996). Amplitude reductions associated with repetitive exposure to similar stimuli have theoretically been related to top-down regulation of sensory networks, or to alterations in the neuro-architecture of systems needed for task execution (Keil, in press). For example, Desimone (1996) suggested that in learning and memory, top-down modulatory input from prefrontal cortex may alter sensory stimulus processing in both a short-term and long-term manner, changing the network architecture underlying a representation. According to Desimone (1996), the neuronal mechanisms related to these top-down modulations may include a variety of processes, such as refinement for learned representations (repetition suppression), increase of amplitude for behaviorally relevant information (enhancement), and continuation of sensory neuronal response after stimulus termination (delay activity). From animal work, it is known that oscillatory activity is capable of mediating these processes (Dumenko 1995; Freeman 1994), allowing for immediate changes as well as 

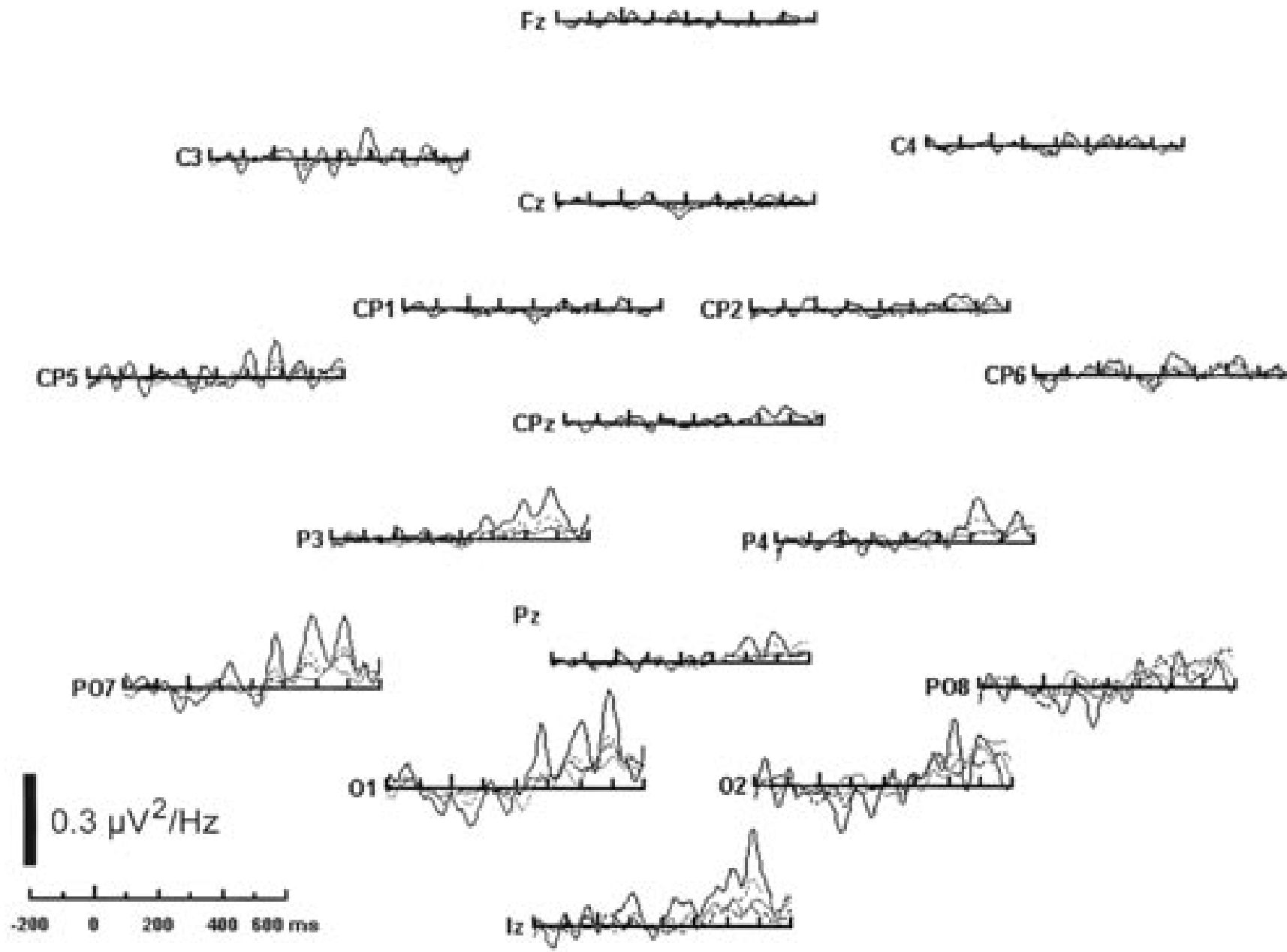

Figure 5. Baseline-corrected time series of induced EEG spectral power changes (45-70 Hz) recorded from subject 2, shown at a subset of the 129-electrode array. Line styles represent different sessions. Black solid: session 1; gray dashed: session 2; black dashed: session 3; gray solid: session 4.

long-term plasticity. Hence, a complete picture of plastic changes during repeated exposure to similar tasks should take oscillatory activity into account (Keil et al. 2001a).

To summarize, we report high temporal stability in measures of induced GBA, both in terms of topography and time course. GBA modulations operationalized as a mean within a time-frequency range thus seem to meet basic requirements for reliability in repeated measures. A linear decrease of GBA amplitude with increasing task familiarity also suggests that GBA modulations reflect meaningful neuronal processes associated with cognitive processing.

\section{References}

Basar, E., Basar-Eroglu, C., Karakas, S. and Schürmann, M. Gamma, alpha, delta, and theta oscillations govern cogni- tive processes. Int. J. Psychophysiol., 2001, 39: 241-248.

Bertrand, O., Bohorquez, J. and Pernier, J. Time-frequency digital filtering based on an invertible wavelet transform: an application to evoked potentials. IEEE Trans. Biomed. Eng., 1994, 41: 77-88.

Desimone, R. Neural mechanisms for visual memory and their role in attention. Proc. Natl. Acad. Sci. USA, 1996, 93: 13494-13499.

Dumenko, V.N. Dynamic shifts in the parameters of the traditional frequency range of the EEG during learning in dogs. Neurosci. Behav. Physiol., 1995, 25: 403-412.

Freeman, W.J. Role of chaotic dynamics in neural plasticity. Prog. Brain Res., 1994, 102: 319-333.

Gruber, T., Muller, M.M. and Keil, A. Modulation of induced gamma band responses in a perceptual learning task in the human EEG. J. Cogn. Neurosci., 2002, 14: 732-744.

Gruber, T., Müller, M.M., Keil, A. and Elbert, T. Selective visual-spatial attention alters induced gamma band responses in the human EEG. Clin. Neurophysiol., 1999, 110: 
2074-2085.

Hauk, O., Keil, A., Elbert, T. and Müller, M.M. Comparison of data transformation procedures to enhance topographical accuracy in time series analysis of the human EEG. J. Neurosci. Methods, 2002,113: 111-122.

Herrmann, C.S., Mecklinger, A. and Pfeifer, E. Gamma responses and ERPs in a visual classification task. Clin. Neurophysiol., 1999, 110: 636-642.

Juergens, E., Guettler, A. and Eckhorn, R. Visual stimulation elicits locked and induced gamma oscillations in monkey intracortical- and EEG-potentials, but not in human EEG. Exp. Brain Res., 1999, 129: 247-259.

Junghöfer, M., Elbert, T., Tucker, D.M. and Rockstroh, B. Statistical control of artifacts in dense array EEG/MEG studies. Psychophysiology, 2000, 37: 523-532.

Keil, A. The role of human prefrontal cortex in motivated perception and behavior: A macroscopic perspective. In: S. Otani (Ed.), Prefrontal Cortex: From Synaptic Plasticity to Cognition. Kluwer, New York, in press.

Keil, A., Gruber, T. and Müller, M.M. Functional correlates of macroscopic high-frequency brain activity in the human visual system. Neurosci. Biobehav. Rev., 2001a, 25: 527-534.

Keil, A., Müller, M.M., Gruber, T., Stolarova, M., Wienbruch, C. and Elbert, T. Effects of emotional arousal in the cerebral hemispheres: a study of oscillatory brain activity and event-related potentials. Clin. Neurophysiol., 2001b, 112: 2057-2068.

Keil, A., Müller, M.M., Gruber, T., Wienbruch, C. and Elbert, T. Human large-scale oscillatory brain activity during an operant shaping procedure. Brain Res. Cogn. Brain Res., 2001c, 12: 397-407.

Keil, A., Müller, M.M., Ray, W.J., Gruber, T. and Elbert, T. Human gamma band activity and perception of a gestalt. J. Neurosci., 1999, 19: 7152-7161.

Müller, M.M., Gruber, T. and Keil, A. Modulation of induced gamma band activity in the human EEG by attention and visual information processing. Int. J. Psychophysiol., 2000, 38: 283-299.

Sannita, W.G. Stimulus-specific oscillatory responses of the brain: a time/frequency-related coding process. Clin. Neurophysiol., 2000, 111: 565-583.

Sinkkonen, J., Tiitinen, H. and Naatanen, R. Gabor filters: an informative way for analysing event-related brain activity. J. Neurosci. Methods, 1995, 56: 99-104.

Slobounov, S., Tutwiler, R., Slobounova, E., Rearick, M. and Ray, W. Human oscillatory brain activity within gamma band $(30-50 \mathrm{~Hz})$ induced by visual recognition of non-stable postures. Brain Res. Cogn. Brain Res., 2000, 9: 177-192.

Tallon-Baudry, C. and Bertrand, O. Oscillatory gamma activity in humans and its role in object representation. Trends Cogn. Sci., 1999, 3: 151-162.

Tallon-Baudry, C., Bertrand, O., Delpuech, C. and Pernier, J. Oscillatory gamma-band $(30-70 \mathrm{~Hz})$ activity induced by a visual search task in humans. J. Neurosci., 1997, 17: 722-734.

Yordanova, J., Banaschewski, T., Kolev, V., Woerner, W. and Rothenberger, A. Abnormal early stages of task stimulus processing in children with attention-deficit hyperactivity disorder-evidence from event-related gamma oscillations. Clin. Neurophysiol., 2001, 112: 1096-1108. 